\title{
Selective NMR Measurements of Homonuclear Scalar Couplings in Isotopically Enriched Solids
}

\section{Supplementary Information}

Sylvian Cadars, Anne Lesage, Niklas Hedin, ${ }^{\dagger}$ Bradley F. Chmelka, ${ }^{\dagger}$ and Lyndon Emsley*

Laboratoire de Chimie (UMR 5182 CNRS/ENS Lyon), Laboratoire de Recherche Conventionné du CEA (DSV 23V / DSM 0432), Ecole Normale Supérieure de Lyon, Lyon, France.

${ }^{\dagger}$ Department of Chemical Engineering, University of California, Santa Barbara, California 93106, U.S.A.

RECEIVED DATE: 12 May 2006.

* to whom correspondence should be addressed. E-mail: lyndon.emsley@ens-lyon.fr

TITLE RUNNING HEAD: $J$-Couplings in Enriched Solids

CORRESPONDING AUTHOR FOOTNOTE

Prof. Lyndon Emsley

Laboratoire de Chimie

Ecole Normale Supérieure de Lyon

46 allée d'Italie, 69364 Lyon cedex 07, France

Phone : +33472728486

Fax : +33472728860

E-mail : 1yndon.emsley@ens-lyon.fr 

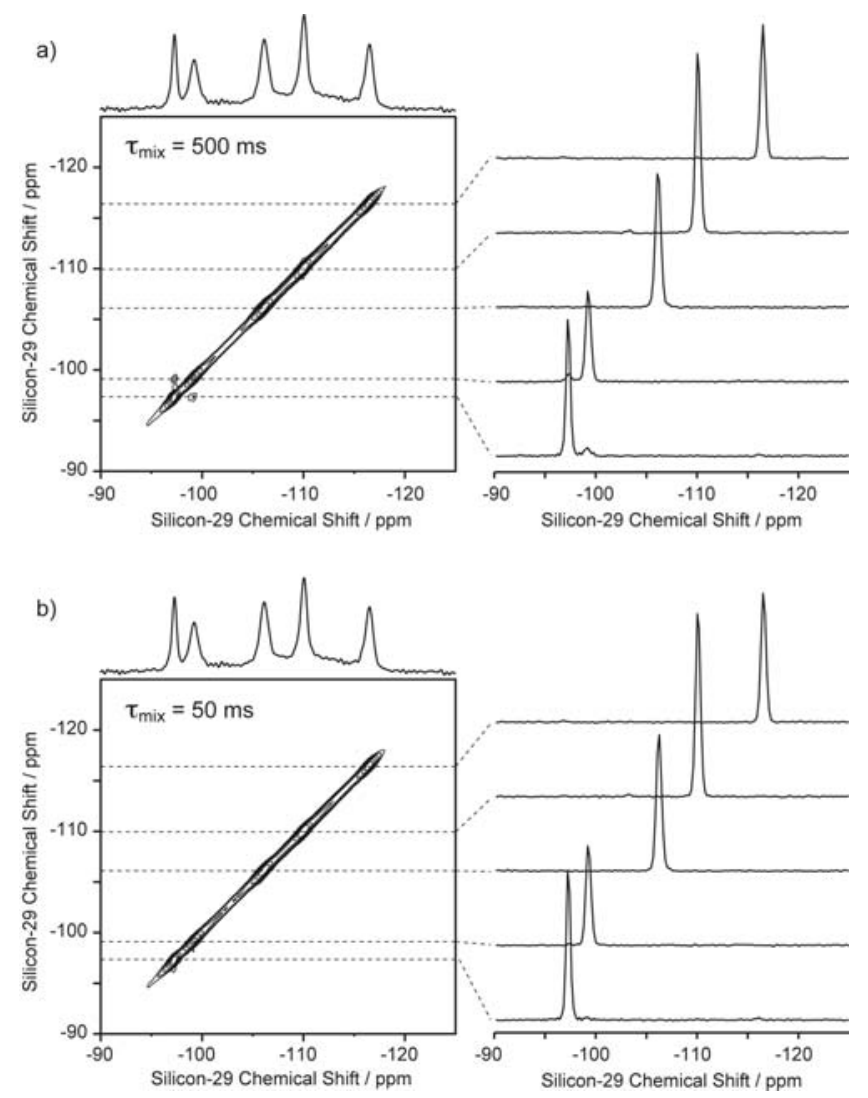

LEGEND: Spin-diffusion spectra are shown, which were carried out in similar MAS (10 $\mathrm{kHz}$ ), temperature $(298 \mathrm{~K})$ and heteronuclear decoupling (SPINAL64 at $90 \mathrm{kHz}$ during indirect and direct detection periods) conditions as the $J$-coupling measurements whose results are shown in Figure 7. They show no or very small correlation peaks, indicating that the long $z$-filers used for the $J$-coupling measurements in the layered silicate do not have any impact on the measured coupling constants. Both experiments were acquired within 8 transients, using $128 t_{1}$ increments, and a recycling delay of $3 \mathrm{~s}$.

(a) Mixing time of $500 \mathrm{~ms}$, without heteronuclear decoupling. Slight magnetization exchange is observed only between the $Q^{3}$ sites. However, it is small enough for the magnetization transfer, which may occur during the $z$-filter subsequent to the selective DQ-filter in the $J$ coupling measurements, to have negligible effect on the measured $J$-coupling value.

(b) Mixing time of $50 \mathrm{~ms}$, without heteronuclear decoupling. No cross peak is observed in this case, indicating that no spin diffusion occurs during a $z$-filter of comparable duration. 